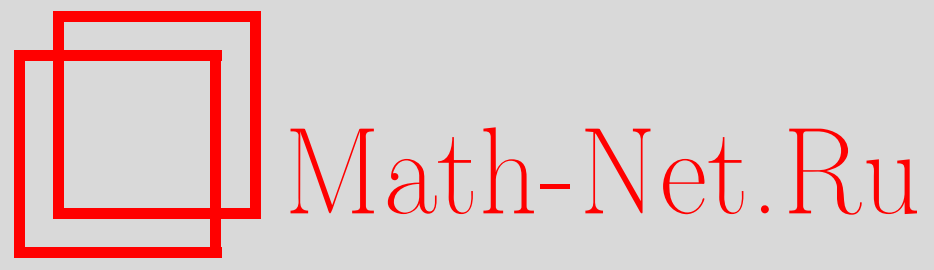

Л. А. Сахнович, О спектральной теории одного класса канонических дифференциальных систем, Функи. анализ и его прил., 2000, том 34, выпуск 2, 50-62

DOI: https://doi.org/10.4213/faa295

Использование Общероссийского математического портала Math$\mathrm{Net.Ru}$ подразумевает, что вы прочитали и согласны с пользовательским соглашением

http://www . mathnet.ru/rus/agreement

Параметры загрузки:

IP : 54.80 .97 .219

26 апреля 2023 г., 15:34:26

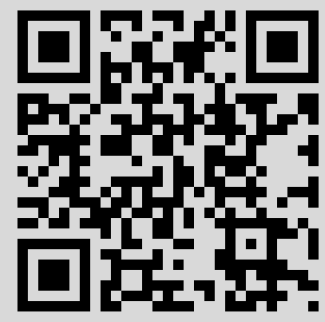


Функииональный анализ и его приложения

2000, т. 34, вып. 2, с. 50-62

УДК 517.9

\title{
О спектраљьнй теории одного класса канонических дифференциальных систем
}

\author{
(c) 2000. Л. А. САхнович
}

В известной статье М. Г. Крейна [1] исследована система дифференциальных уравнений

где

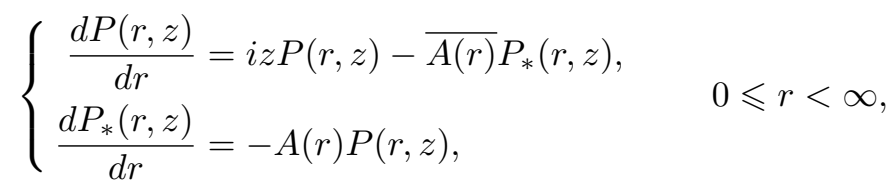

$$
P(0, z)=P_{*}(0, z)=1 .
$$

Функция $A(r)$ предполагается непрерывной.

Монотонно возрастающая функция $\tau(\lambda)(-\infty<\lambda<\infty)$ называется cneктральной для системь (0.1), если для любой финитной функции $f(r) \in L_{2}(0, \infty)$ справедливо равенство

где

$$
\int_{0}^{\infty}|f(r)|^{2} d r=\int_{-\infty}^{\infty}|F(\lambda)|^{2} d \tau(\lambda)
$$

$$
F(\lambda)=\int_{0}^{\infty} \overline{P(r, \lambda)} f(r) d r .
$$

В работе М. Г. Крейна [1] дано полное решение обратной спектральной задачи для системы $(0.1)$, т.е. дана процедура построения $A(r)$, если известна спектральная функция $\tau(\lambda)$. K сожалению, эти важные результаты опубликованы без доказательства. Мы доказали все утверждения Крейна, сформулированные в статье [1], и перенесли соответствующие результаты на широкий класс канонических дифференциальных систем. Полученные в этом направлении результаты частично опубликованы в статье [2]. Данная статья посвящена доказательству и обобщению важных утверждений Крейна из [1], относящихся к связи между спектральной функцией $\tau(\lambda)$ системы $(0.1)$ с пределом

и интегралом

$$
\mathscr{P}(z)=\lim _{r \rightarrow \infty} P_{*}(r, z)
$$

$$
\left.K(z)=\int_{0}^{\infty}|P(r, z)|^{2} d r \quad \operatorname{Im} z>0\right) .
$$

\section{$\S 1$. Основные понятия}

1.1. Рассмотрим каноническую систему дифференциальных уравнений

$$
\frac{d Y}{d x}=i z J \mathscr{H}(x) Y, \quad 0 \leqslant x<\infty,
$$


где $\mathscr{H}(x)$ - комплекснозначная непрерывная неотрицательная $2 m \times 2 m$-матрица-функция,

$$
J=\left[\begin{array}{cc}
0 & E_{m} \\
E_{m} & 0
\end{array}\right],
$$

а $Y(x, z)$ - вектор-столбец размера $2 m \times 1$. Будем далее предполагать, что

$$
J \mathscr{H}(x)=T(x) M T^{-1}(x),
$$

где

$$
M=\left[\begin{array}{cc}
D & 0 \\
0 & 0
\end{array}\right], \quad D=\operatorname{diag}\left\{d_{1}, \ldots, d_{m}\right\}, \quad d_{k}=\text { const }>0 .
$$

Как известно (см. [2, 3]), к системам вида (1.1)-(1.3) могут быть сведены как классические задачи (система Крейна, система типа Дирака, матричное уравнение Штурма-Лиувилля, матричное уравнение струны), так и ряд неклассических спектральных задач.

Согласно теореме В. П. Потапова [4, гл. 1, §2] о J-неотрицательных матрицах, без ограничения общности можно считать, что матрица $T(x)$ в формуле (1.2) удовлетворяет соотношению

$$
T(x) j T^{*}(x)=J
$$

где

$$
j=\left[\begin{array}{cc}
E_{m} & 0 \\
0 & -E_{m}
\end{array}\right] .
$$

Мы будем предполагать далее, что матрица-функция $T(x)$ непрерывно дифференцируема.

1.2. Введем, кроме того, матричное решение $w(x, z)$ системы

$$
\frac{d w}{d x}=i z J \mathscr{H}(x) w(x, z), \quad w(0, z)=E_{2 m},
$$

а также пространство $L^{2}(\mathscr{H})$ векторов-функций со скалярным произведением

$$
(g, h)=\int_{0}^{l} h^{*}(x) \mathscr{H}(x) g(x) d x,
$$

где $l$ - любое конечное число.

Монотонно возрастающую $m \times m$-матрицу-функцию $\tau(\lambda)(-\infty<\lambda<\infty)$ будем называть спектральной матрицей-функиией системь (1.1), если оператор

$$
V g=\int_{0}^{l}\left[0, E_{m}\right] w^{*}(x, \lambda) \mathscr{H}(x) g(x) d x=F(\lambda)
$$

изометрически отображает пространство $L^{2}(\mathscr{H})$ в $L^{2}(\tau)$ (см. [3]).

В пространстве $L^{2}(\tau)$ скалярное произведение определено формулой

$$
\left(F_{1}, F_{2}\right)=\int_{-\infty}^{\infty} F_{2}^{*}(\lambda)[d \tau(\lambda)] F_{1}(\lambda) .
$$

В силу изометричности оператора $V$ выполняется равенство Парсеваля

$$
\int_{0}^{l} g^{*}(x) \mathscr{H}(x) g(x) d x=\int_{-\infty}^{\infty} F^{*}(\lambda)[d \tau(\lambda)] F(\lambda) .
$$


Пусть

$$
W(x, z)=T^{-1}(x) w(x, z) T(0) .
$$

Дифференцируя (1.8) и учитывая (1.2), получаем

$$
\frac{d W(x, z)}{d x}=i z M W(x, z)+A(x) W(x, z),
$$

где

$$
A(x)=-T^{-1}(x) \frac{d T(x)}{d x} .
$$

Из соотношений (1.4) и (1.10) следует, что

$$
A(x) j=-j A^{*}(x) .
$$

Запишем матрицы $W(x, z), A(x)$ и $S(x)=T^{-1}(x)$ в блочном виде:

$$
\begin{gathered}
W(x, z)=\left[\begin{array}{ll}
W_{11}(x, z) & W_{12}(x, z) \\
W_{21}(x, z) & W_{22}(x, z)
\end{array}\right] \\
A(x)=\left[\begin{array}{ll}
A_{11}(x) & A_{12}(x) \\
A_{21}(x) & A_{22}(x)
\end{array}\right], \quad S(x)=\left[\begin{array}{ll}
S_{11}(x) & S_{12}(x) \\
S_{21}(x) & S_{22}(x)
\end{array}\right],
\end{gathered}
$$

где все блоки имеют размер $m \times m$. В силу (1.11) верны равенства

$$
A_{11}(x)=-A_{11}^{*}(x), \quad A_{21}(x)=A_{12}^{*}(x) .
$$

Условиями (1.2) и (1.4) матрица $T(x)$ определяется неоднозначно. Учитывая этот факт, выберем матрицу $T(x)$ так, что

$$
A_{22}(x)=0 .
$$

Важную роль в дальнейшем играют матрицы-функции

$$
\begin{aligned}
& P_{1}(x, z)=W_{11}(x, z) S_{12}(0)+W_{12}(x, z) S_{22}(0), \\
& P_{2}(x, z)=W_{21}(x, z) S_{12}(0)+W_{22}(x, z) S_{22}(0) .
\end{aligned}
$$

В силу (1.9) матрицы-функции $P_{1}(x, z)$ и $P_{2}(x, z)$ удовлетворяют системе дифференциальных уравнений

$$
\left\{\begin{array}{l}
d P_{1} / d x=i z D P_{1}+A_{11}(x) P_{1}+A_{12}(x) P_{2}, \\
d P_{2} / d x=A_{21}(x) P_{1}, \quad 0 \leqslant x,
\end{array}\right.
$$

а также следующим условиям на левом конце:

$$
P_{1}(0, z)=S_{12}(0), \quad P_{2}(0, z)=S_{22}(0) .
$$

Если

$$
m=1, \quad d_{1}=1, \quad A_{11}(x)=0, \quad S_{12}(0)=S_{22}(0)=1,
$$

то система (1.16) совпадает с системой Крейна (0.1). Если $m>1$ и $d_{j} \neq d_{k}$ $(j \neq k)$, то эта система приводит к новым спектральным задачам, которые представляют интерес как с теоретической, так и с прикладной точек зрения. Система (1.16) является главным объектом исследования в данной статье. 
1.3. Перепишем формулы (1.5), (1.6) в терминах системы (1.16). Учитывая (1.8), имеем

$$
V g=\int_{0}^{l}\left[0, E_{m}\right] S^{*}(0) W^{*}(x, \lambda) T^{*}(x) \mathscr{H}(x) g(x) d x .
$$

Пользуясь формулами (1.2), (1.4), преобразуем (1.19) к виду

$$
F(\lambda)=V g=\int_{0}^{l}\left[0, E_{m}\right] S^{*}(0) W^{*}(x, \lambda) M T^{-1}(x) g(x) d x .
$$

Здесь мы учли, что из (1.4) следует равенство

$$
T^{*}(x) J T(x)=j .
$$

Представим теперь вектор $g(x)$ в виде

$$
g(x)=\operatorname{col}\left[g_{1}(x), g_{2}(x)\right],
$$

где $g_{k}(x)$ - векторы-столбцы размера $m \times 1$. Тогда из (1.20) вытекает равенство

$$
F(\lambda)=\int_{0}^{l} P_{1}^{*}(x, \lambda) D f(x) d x
$$

где $P_{1}(x, u)$ определяется формулой (1.14), а

$$
f(x)=S_{11}(x) g_{1}(x)+S_{12}(x) g_{2}(x) .
$$

Соотношение (1.7) принимает вид

$$
\int_{-\infty}^{\infty} F^{*}(\lambda)[d \tau(\lambda)] F(\lambda)=\int_{0}^{l} f^{*}(x) D f(x) d x .
$$

Таким образом, спектральная матрица-функция системы (1.16) определяется соотношениями (1.21), (1.22).

Если выполняются условия (1.18), то формулы (1.21) и (1.22) совпадают с формулами Крейна (0.2) и (0.3).

\section{$\S 2$. Обобщение и доказательство двух теорем М. Г. Крейна}

Основной целью данного параграфа является доказательство следующих теорем.

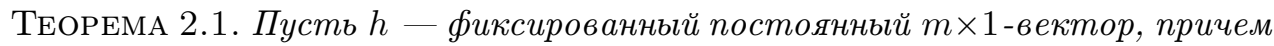
$h \neq 0$. Следующие утверждения эквивалентны:

1) интеграл

$$
K_{h}(z)=\int_{0}^{\infty} h^{*} P_{1}^{*}(x, z) D P_{1}(x, z) h d x
$$

сходится по крайней мере для одного $z_{0}\left(\operatorname{Im} z_{0}>0\right)$;

2) норма вектора $P_{2}(x, z) h(0 \leqslant x<\infty)$ ограничена по крайней мере для одного $z_{0}\left(\operatorname{Im} z_{0}>0\right)$;

3) на любом ограниченном замкнутом множестве точек z открьтой полуплоскости $\operatorname{Im} z>0$ интеграл (2.1) сходится равномерно;

4) на любом ограниченном замкнутом множестве точек z открытой полуплоскости $\operatorname{Im} z>0$ существует равномерно сходящийся предел

$$
\mathscr{P}_{h}(z)=\lim _{x \rightarrow \infty} P_{2}(x, z) h .
$$


ТеОрема 2.2. Следующие утверждения эквивалентны:

1) справедливо неравенство

$$
\int_{-\infty}^{\infty} \frac{\log \operatorname{det} \tau^{\prime}(\lambda)}{1+\lambda^{2}} d \lambda>-\infty
$$

где $\tau(\lambda)$ - спектральная $m \times m$-матрица-функиия системы (1.16), (1.17);

2) на любом ограниченном замкнутом множестве точек z полуплоскости $\operatorname{Im} z>0$ существует равномерно сходящийся предел

$$
\mathscr{P}(z)=\lim _{x \rightarrow+\infty} P_{2}(x, z), \quad \operatorname{Im} z>0 .
$$

ЗАмЕЧАниЕ 2.1. Если выполнены условия (1.18), то теоремы 2.1 и 2.2 совпадают с теоремами Крейна, сформулированными в статье [1].

ДОКАЗАТЕЛЬСТВО ТЕОРЕМЫ 2.1. Дифференцируя равенство

$$
P(x, z, \xi)=P_{2}^{*}(x, z) P_{2}(x, \xi)-P_{1}^{*}(x, z) P_{1}(x, \xi)
$$

по переменной $x$ и принимая во внимание соотношения (1.12), (1.13) и (1.16), выводим равенство

$$
\frac{d P}{d x}=i(\bar{z}-\xi) P_{1}^{*}(x, z) D P_{1}(x, \xi) .
$$

Согласно (1.4), имеем

$$
S^{*}(x) j S(x)=J .
$$

В частности, из (2.4) следует, что

$$
S_{12}^{*}(0) S_{12}(0)=S_{22}^{*}(0) S_{22}(0) .
$$

В силу $(1.17),(2.2)$ и $(2.5)$ верно равенство $P(0, z, \xi)=0$. Тогда из (2.3) получаем, что

$$
P_{2}^{*}(x, z) P_{2}(x, \xi)-P_{1}^{*}(x, z) P_{1}(x, \xi)=i(\bar{z}-\xi) \int_{0}^{x} P_{1}^{*}(t, z) D P_{1}(t, \xi) d t .
$$

Если $\xi=z$, то из (2.6) следует формула

$$
P_{2}^{*}(x, z) P_{2}(x, z)-P_{1}^{*}(x, z) P_{1}(x, z)=2 \operatorname{Im} z \int_{0}^{x} P_{1}^{*}(t, z) D P_{1}(t, z) d t .
$$

Введем теперь интеграл

$$
K_{h}(z, \xi, R)=h^{*} \int_{0}^{R} P_{1}^{*}(x, z) D P_{1}(x, \xi) d x h .
$$

Согласно (2.7), справедливо неравенство

$$
h^{*} P_{2}^{*}(x, z) P_{2}(x, z) h \geqslant 2 \operatorname{Im} z K_{h}(z, z, R), \quad x \geqslant R .
$$

Функция $K_{h}(z, z, R)$ монотонно возрастает с ростом $R$ и имеет конечный или бесконечный предел

$$
\lim _{R \rightarrow \infty} K_{h}(z, z, R)=K_{h}(z) \leqslant \infty .
$$

Пусть $Q$ - замкнутое ограниченное множество точек, принадлежащее открытой полуплоскости $\operatorname{Im} z>0$. Согласно $(2.8)$, последовательность $P_{2}\left(R_{n}, z\right) h$ 
$\left(R_{n} \rightarrow \infty\right)$ равномерно ограничена снизу в области $Q$. Согласно теореме Монтеля, существует равномерный предел

$$
\mathscr{P}_{h}(z)=\lim _{R_{n} \rightarrow \infty} P_{2}\left(R_{n}, z\right) h, \quad z \in Q,
$$

причем

$$
\mathscr{P}_{h}(z) \neq 0, \quad \operatorname{Im} z>0 .
$$

Вообще говоря, возможен случай, когда

$$
\left\|\mathscr{P}_{h}(z)\right\|=\infty .
$$

Мы покажем, что условия теоремы исключают возможность такого случая.

Пусть выполнено условие 1) теоремы. Тогда существует последовательность $P_{1}\left(R_{k}, z\right) h$, такая, что

$$
P_{1}\left(R_{k}, z\right) h \rightarrow 0 .
$$

В этом случае из соотношения (2.7) вытекает, что последовательность $P_{2}\left(R_{k}, z\right) h$ ограничена, т. е. $\left\|\mathscr{P}_{h}(z)\right\|<\infty$.

Итак, мы доказали, что условия 2)-4) следуют из условия 1). Остальные утверждения теоремы доказываются аналогично.

ЗАмЕЧАниЕ 2.2. Пусть одно из условий теоремы 2.1 выполнено. Тогда из (2.7) и (2.10) следует, что

$$
\begin{gathered}
P_{1}(R, z) h \rightarrow 0 \quad(R \rightarrow \infty), \quad \operatorname{Im} z>0, \\
\mathscr{P}_{h}^{*}(z) \mathscr{P}_{h}(z)=\int_{0}^{\infty} h^{*} P_{1}^{*}(x, z) P_{1}(x, z) h d x .
\end{gathered}
$$

ДОКАЗАТЕЛЬСТВо ТЕОРЕМЫ 2.2. Из (1.9), (1.12) и (1.13) вытекает равенство

$$
\frac{d}{d x}\left[W^{*}(x, z) j W(x, z)\right]=i(z-\bar{z}) W^{*}(x, z) M W(x, z) .
$$

Откуда следует, что

$$
W^{*}(x, z) j W(x, z)=j+i(z-\bar{z}) \int_{0}^{x} W^{*}(t, z) M W(t, z) d t .
$$

Значит, верны неравенства

$$
W^{*}(x, z) j W(x, z) \leqslant j, \quad W(x, z) j W^{*}(x, z) \leqslant j, \quad \operatorname{Im} z>0 .
$$

Наряду с $P_{1}(x, z)$ и $P_{2}(x, z)$ введем матрицы-функции

$$
\begin{aligned}
& \widehat{P}_{1}(x, z)=W_{11}(x, z) S_{11}(0)+W_{12}(x, z) S_{21}(0), \\
& \widehat{P}_{2}(x, z)=W_{21}(x, z) S_{11}(0)+W_{22}(x, z) S_{21}(0) .
\end{aligned}
$$

Второе из неравенств (2.13) можно записать в виде

$$
W(x, z) S(0) J S^{*}(0) W^{*}(x, z) \leqslant j, \quad \operatorname{Im} z>0 .
$$

Мы учли здесь, что, согласно (1.4), выполняется равенство

$$
S(0) J S^{*}(0)=j \text {. }
$$


В силу $(1.14),(1.15)$ и $(2.14),(2.15)$ из неравенства (2.16) вытекает, что

$$
\left[\begin{array}{ll}
\widehat{P}_{1}(x, z) & P_{1}(x, z) \\
\widehat{P}_{2}(x, z) & P_{2}(x, z)
\end{array}\right] J\left[\begin{array}{ll}
\widehat{P}_{1}^{*}(x, z) & \widehat{P}_{2}^{*}(x, z) \\
P_{1}^{*}(x, z) & P_{2}(x, z)
\end{array}\right] \leqslant j .
$$

Из соотношения (2.17) выводим неравенство

$$
P_{2}(x, z) \widehat{P}_{2}^{*}(x, z)+\widehat{P}_{2}(x, z) P_{2}^{*}(x, z) \leqslant-E_{m}, \quad \operatorname{Im} z>0 .
$$

Предположим сначала, что выполнено условие 2) теоремы. Тогда, как показано в статье [2], существует и предел

$$
\widehat{\mathscr{P}}(z)=\lim _{x \rightarrow+\infty} \widehat{P}_{2}(x, z), \quad \operatorname{Im} z>0 .
$$

Переходя в неравенстве $(2.18)$ к пределу при $x \rightarrow+\infty$, получаем

$$
\mathscr{P}(z) \widehat{\mathscr{P}}^{*}(z)+\widehat{\mathscr{P}}(z) \mathscr{P}^{*}(z) \leqslant-E_{m}, \quad \operatorname{Im} z>0 .
$$

Из (2.9) следует, что

$$
\operatorname{det} \mathscr{P}(z) \neq 0, \quad \operatorname{Im} z>0 .
$$

Введем матрицу-функцию

$$
v(z)=-i \mathscr{P}^{-1}(z) \widehat{\mathscr{P}}(z), \quad \operatorname{Im} z>0 .
$$

В силу (2.20) имеем

$$
\frac{v(z)-v^{*}(z)}{i}=-\mathscr{P}^{-1}(z)\left[\widehat{\mathscr{P}}(z) \mathscr{P}^{*}(z)+\mathscr{P}(z) \widehat{\mathscr{P}}^{*}(z)\right] \mathscr{P}^{*-1}(z), \quad \operatorname{Im} z>0 .
$$

Согласно соотношениям (2.19) и (2.21), справедливо неравенство

$$
\frac{v(z)-v^{*}(z)}{i} \geqslant \mathscr{P}^{-1}(z) \mathscr{P}^{*-1}(z) .
$$

Таким образом, матрица-функция $v(z)$ принадлежит классу Неванлинны. По теореме В. И. Смирнова (см. [6]) элементы матрицы $v(z)$ принадлежат классу Харди $H_{\delta}(0<\delta<1)$. Тогда, согласно неравенству $(2.22)$, элементы матрицы $\mathscr{P}^{-1}(z)$ также принадлежат классу Харди $H_{\delta}$. Значит, почти всюду существуют предельные значения

$$
v_{+}(\lambda)=\lim _{\varepsilon \rightarrow+0} v(\lambda+i \varepsilon), \quad \mathscr{P}_{+}^{-1}(\lambda)=\lim _{\varepsilon \rightarrow+0} \mathscr{P}^{-1}(\lambda+i \varepsilon), \quad \lambda=\bar{\lambda} .
$$

Заметим, что

$$
\operatorname{det} \mathscr{P}_{+}^{-1}(\lambda)=\lim _{\varepsilon \rightarrow+0} \operatorname{det} \mathscr{P}^{-1}(\lambda+i \varepsilon), \quad \lambda=\bar{\lambda} .
$$

Так как элементы матрицы $\mathscr{P}^{-1}(z)$ принадлежат классу $H_{\delta}$, то $\operatorname{det} \mathscr{P}^{-1}(z)$ принадлежит классу $A$ (см. [6]), т. е. почти всюду на оси $-\infty<\lambda<\infty$ верны соотношения

$$
\begin{gathered}
\operatorname{det} \mathscr{P}_{+}^{-1}(\lambda) \neq 0, \\
\int_{-\infty}^{\infty} \frac{\log \left|\operatorname{det} \mathscr{P}_{+}^{-1}(\lambda)\right|^{2}}{1+\lambda^{2}} d \lambda>-\infty .
\end{gathered}
$$


В статье [2] установлена формула

$$
v(z)=\alpha+\int_{-\infty}^{\infty}\left(\frac{1}{u-z}-\frac{u}{1+u^{2}}\right) d \tau(u),
$$

связывающая введенную функцию $v(z)$ и спектральную матрицу-функцию $\tau(u)$ системы (1.16), (1.17).

Из представления (2.25) и формулы обращения Стилтьеса вытекает, что почти всюду верно равенство

$$
\frac{v_{+}(\lambda)-v_{+}^{*}(\lambda)}{i}=2 \pi \tau^{\prime}(\lambda) .
$$

В силу формул $(2.22)$ и (2.26) имеем

$$
\tau^{\prime}(\lambda) \geqslant \frac{1}{2 \pi} \mathscr{P}_{+}^{-1}(\lambda) \mathscr{P}_{+}^{*-1}(\lambda) .
$$

Из соотношений $(2.24)$ и (2.27) следует неравенство

$$
\int_{-\infty}^{\infty} \frac{\log \operatorname{det} \tau^{\prime}(\lambda)}{1+\lambda^{2}} d \lambda>-\infty .
$$

Таким образом, доказано, что из выполнения условия 2) теоремы вытекает выполнение условия 1).

Докажем теперь, что из выполнения условия 1) вытекает выполнение условия 2).

Для этого введем функцию

$$
K_{R}(z, \xi)=\int_{0}^{R} P_{1}^{*}(x, z) D P_{1}(x, \xi) d x .
$$

В силу равенства Парсеваля (1.22) и формулы (2.28) верно соотношение

$$
\int_{-\infty}^{\infty} K_{R}^{*}(\lambda, \xi)[d \tau(\lambda)] K_{R}(\lambda, \xi)=\int_{0}^{R} P_{1}^{*}(x, \xi) D P_{1}(x, \xi) d x .
$$

Сопоставляя (2.28) и (2.29), получаем равенство

$$
\int_{-\infty}^{\infty} K_{R}^{*}(\lambda, \xi)[d \tau(\lambda)] K_{R}(\lambda, \xi)=K_{R}(\xi, \xi) .
$$

Из соотношения (2.28) вытекает, что

$$
K_{R}(z, \xi)>0 .
$$

Из формулы Лагранжа и равенства (2.28) следует, что

Положим

$$
K_{R}(z, \xi)=\frac{P_{2}^{*}(R, z) P_{2}(R, \xi)-P_{1}^{*}(R, z) P_{1}(R, \xi)}{i(\bar{z}-\xi)} .
$$

$$
Q_{R}(z, \xi)=P_{2}^{*}(R, z) P_{2}(R, \xi)-P_{1}^{*}(R, z) P_{1}(R, \xi) .
$$

В силу формул (2.31) и (2.32) верно неравенство

$$
K_{R}(\xi, \xi)=\frac{Q_{R}(\xi, \xi)}{i(\bar{\xi}-\xi)}>0 .
$$


Перепишем соотношение (2.30) в следующем виде:

где

$$
\int_{-\infty}^{\infty} \frac{\widetilde{Q}_{R}^{*}(\lambda, \xi)[d \tau(\lambda)] \widetilde{Q}_{R}(\lambda, \xi)}{|\lambda-\xi|^{2}}=\frac{Q_{R}^{-1}(\xi, \xi)}{i(\bar{\xi}-\xi)}
$$

$$
\widetilde{Q}_{R}(\lambda, \xi)=Q_{R}(\lambda, \xi) Q_{R}^{-1}(\xi, \xi) .
$$

Согласно (2.35), выполняется неравенство

$$
\frac{Q_{R}^{-1}(\xi, \xi)}{i(\bar{\xi}-\xi)} \geqslant \int_{-\infty}^{\infty} \frac{\widetilde{Q}_{R}^{*}(\lambda, \xi) \tau^{\prime}(\lambda) \widetilde{Q}_{R}(\lambda, \xi)}{|\lambda-\xi|^{2}} d \lambda,
$$

откуда следует, что

$$
\ln \operatorname{det}\left[\frac{1}{\pi} \frac{Q_{R}^{-1}(\xi, \xi)}{i(\bar{\xi}-\xi)}\right] \geqslant \ln \operatorname{det}\left[\frac{1}{\pi} \int_{-\infty}^{\infty} \frac{\widetilde{Q}_{R}^{*}(\lambda, \xi) \tau^{\prime}(\lambda) \widetilde{Q}_{R}(\lambda, \xi)}{|\lambda-\xi|^{2}} d \lambda\right] .
$$

Воспользуемся известным неравенством (см. [7, гл. II, §6])

$$
\ln \operatorname{det} \frac{1}{\pi} \int_{-\infty}^{\infty} \frac{g(\lambda)}{1+\lambda^{2}} d \lambda \geqslant \frac{1}{\pi} \int_{-\infty}^{\infty} \frac{\ln \operatorname{det} g(\lambda)}{1+\lambda^{2}} d \lambda .
$$

Здесь предполагается, что $m \times m$-матрица $g(\lambda)$ положительна. При $\xi=i$ из неравенств (2.37), (2.38) выводим, что

$$
\begin{aligned}
\operatorname{det}\left[\frac{1}{2 \pi} Q_{R}^{-1}(i, i)\right] \geqslant & \exp \frac{2}{\pi} \int_{-\infty}^{\infty} \frac{\ln \left|\operatorname{det} \widetilde{Q}_{R}(\lambda, i)\right|}{1+\lambda^{2}} d \lambda \\
& \times \exp \left[\frac{1}{\pi} \int_{-\infty}^{\infty} \frac{\ln \operatorname{det} \tau^{\prime}(\lambda)}{1+\lambda^{2}} d \lambda\right] .
\end{aligned}
$$

Так как $\left|\operatorname{det} \widetilde{Q}_{R}(\lambda, i)\right|$ является субгармонической функцией, то

$$
\frac{1}{\pi} \int_{-\infty}^{\infty} \frac{\ln \operatorname{det}\left|\widetilde{Q}_{R}(\lambda, i)\right|}{1+\lambda^{2}} d \lambda \geqslant \ln \left|\operatorname{det} \widetilde{Q}_{R}(i, i)\right|=0 .
$$

Тогда в силу (2.39) имеем

$$
\operatorname{det}\left[\frac{1}{2 \pi} Q_{R}^{-1}(i, i)\right] \geqslant \exp \frac{1}{\pi} \int_{-\infty}^{\infty} \frac{\ln \operatorname{det} \tau^{\prime}(\lambda)}{1+\lambda^{2}} d \lambda .
$$

Матрица $K_{R}(i, i)$ положительна и монотонно возрастает. Кроме того, из условия 1$)$, соотношения $(2.34)$ и неравенства $(2.40)$ следует, что $\operatorname{det} K_{R}(i, i)$ при $R \rightarrow \infty$ имеет конечный предел. Значит, матрица $K_{R}(i, i)$ также имеет конечный предел при $R \rightarrow \infty$. Из теоремы 2.1 следует теперь выполнение условия 2) доказываемой теоремы. Теорема доказана.

ЗАмЕчание 2.3. Как показано в статье [2], справедливо неравенство

$$
\int_{0}^{\infty}\left[\widehat{P}_{1}(x, z)-i P_{1}(x, z) v(z)\right]^{*} D\left[\widehat{P}_{1}(x, z)-i P_{1}(x, z) v(z)\right] d x<\infty, \quad \operatorname{Im} z>0 .
$$

Таким образом, введенная формулой (2.20) матрица-функция $v(z)$ является аналогом классической функции Вейля-Титчмарша. 
ЗАмЕчАниЕ 2.4. В статье [2] и в книге [3] содержится метод решения обратной задачи для системы (1.16), т.е. дан метод восстановления коэффициентов $A_{i j}(x)$ по известной спектральной матрице-функции.

\section{§3. Мультипликативное представление для $\mathscr{P}(\lambda)$}

В данном параграфе мы докажем, что в соотношении (2.27) выполняется знак равенства, т. е.

$$
\tau^{\prime}(\lambda)=\frac{1}{2 \pi} \mathscr{P}_{+}^{-1}(\lambda) \mathscr{P}_{+}^{*-1}(\lambda) .
$$

Из соотношения (3.1) мы выведем мультипликативное представление матрицы-функции $\mathscr{P}(\lambda)$.

ТЕОРЕМА 3.1. Пусть $\tau(\lambda)$ является спектральной матрицей-функиией системьл (1.16), (1.17) u

$$
\int_{-\infty}^{\infty} \frac{\ln \operatorname{det} \tau^{\prime}(\lambda)}{1+\lambda^{2}} d \lambda>-\infty .
$$

Тогда справедливо равенство (3.1).

ДокАЗАТЕЛЬСтво. Введем матрицу-функцию

$$
\psi_{R}(z)=Q_{R}(z, i) P_{2}^{-1}(z, i) .
$$

Из формул (2.33)-(2.36) и (3.3) следует, что

$$
\int_{-\infty}^{\infty} \frac{\psi_{R}^{*}(\lambda) \tau^{\prime}(\lambda) \psi_{R}(\lambda)}{1+\lambda^{2}} d \lambda \leqslant \frac{E_{m}}{2} .
$$

Как известно (см., например, $[7$, гл. 3, §2]), из условия (3.2) вытекает факторизуемость матрицы $\tau^{\prime}(\lambda)$, т. е. существует аналитическая в верхней полуплоскости $m \times m$-матрица-функция $\Gamma(z)$, такая, что

$$
\begin{gathered}
\operatorname{det} \Gamma(z) \neq 0, \quad \operatorname{Im} z>0, \\
\Gamma(z) \Gamma^{*}(z)=\tau^{\prime}(\lambda),
\end{gathered}
$$

где

$$
\Gamma(\lambda)=\lim _{\varepsilon \rightarrow+0} \Gamma(\lambda+i \varepsilon) .
$$

В силу (3.6) неравенство (3.4) может быть записано в виде

$$
\int_{-\infty}^{\infty} \frac{\varphi_{R}^{*}(\lambda) \varphi_{R}(\lambda)}{\lambda^{2}+1} d \lambda \leqslant \frac{E_{m}}{2},
$$

где

$$
\varphi_{R}(z)=\Gamma^{*}(z) \psi_{R}(z) .
$$

Матрица-функция $\varphi_{R}^{*}(z)$ является аналитической в верхней полуплоскости и ее элементы, согласно неравенству (3.7), принадлежат классу Харди $H_{2}$. Следовательно, по теореме А. Хинчина и А. Островского (см. [6, гл. 2, §7]) можно в неравенстве (3.7) перейти к пределу при $R \rightarrow \infty$, т. е.

$$
\int_{-\infty}^{\infty} \frac{\varphi_{+}^{*}(\lambda) \Gamma(\lambda) \Gamma^{*}(\lambda) \varphi_{+}(\lambda)}{\lambda^{2}+1} d \lambda \leqslant \frac{E_{m}}{2} .
$$


Здесь приняты следующие обозначения:

$$
\begin{aligned}
\varphi(z) & =\lim _{R \rightarrow \infty} \varphi_{R}(z), & & \operatorname{Im} z>0, \\
\varphi_{+}(\lambda) & =\lim _{\varepsilon \rightarrow+0} \varphi(\lambda+i \varepsilon), & & \lambda=\bar{\lambda} .
\end{aligned}
$$

В силу теоремы 2.2 существует предел

$$
\lim _{R \rightarrow \infty} P_{2}(R, z)=\mathscr{P}(z), \quad \operatorname{Im} z>0 .
$$

Согласно (2.11), верно равенство

$$
\lim _{R \rightarrow \infty} P_{1}(R, z)=0, \quad \operatorname{Im} z>0 .
$$

Значит, учитывая (2.33), (3.3) и (3.8), имеем

$$
\varphi(z)=\mathscr{P}^{*}(z) .
$$

Таким образом, неравенство (3.8) может быть записано в виде

$$
\int_{-\infty}^{\infty} \frac{\mathscr{P}_{+}(\lambda) \Gamma(\lambda) \Gamma^{*}(\lambda) \mathscr{P}_{+}^{*}(\lambda)}{\lambda^{2}+1} d \lambda \leqslant \frac{E_{m}}{2} .
$$

С другой стороны, согласно (2.27), получаем

$$
\int_{-\infty}^{\infty} \frac{\mathscr{P}_{+}(\lambda) \Gamma(\lambda) \Gamma^{*}(\lambda) \mathscr{P}_{+}^{*}(\lambda)}{\lambda^{2}+1} d \lambda \geqslant \frac{1}{2 \pi} \int_{-\infty}^{\infty} \frac{d \lambda}{\lambda^{2}+1} E_{m}=\frac{1}{2} E_{m}
$$

Сравнивая (3.9) и (3.10), выводим равенство

$$
\int_{-\infty}^{\infty} \frac{\mathscr{P}_{+}(\lambda) \Gamma(\lambda) \Gamma^{*}(\lambda) \mathscr{P}_{+}^{*}(\lambda)}{\lambda^{2}+1} d \lambda=\frac{1}{2} E_{m}
$$

Утверждение теоремы теперь следует непосредственно из соотношений (2.27) и $(3.11)$.

ПримеР 3.1. Рассмотрим простейший случай, когда

$$
m=1, \quad A_{i j}(x)=0, \quad S_{12}(0)=S_{22}(0)=1 .
$$

В этом случае

$$
P_{1}(x, z)=e^{i x z}, \quad P_{2}(x, z)=1, \quad \mathscr{P}(z)=1, \quad \tau(\lambda)=\lambda /(2 \pi) .
$$

Теорема 3.2. Пусть $\tau(\lambda)$ - спектральная матрица-функиия системьь (1.16), (1.17) и выполнено условие

$$
\int_{-\infty}^{\infty} \frac{\ln \operatorname{det} \tau^{\prime}(\lambda)}{1+\lambda^{2}} d \lambda>-\infty
$$

Тогда существуют такие матриць $\mathscr{H}(t)$ и $U$, что верно следующее мультипликативное представление:

причем

$$
\sqrt{2 \pi} \mathscr{P}(z)=U \int_{-\infty}^{\curvearrowleft} \exp \left[\frac{1}{2 \pi i} \frac{1+\lambda z}{\lambda-z} \frac{\mathscr{H}(\lambda)}{1+\lambda^{2}} d \lambda\right],
$$

$$
\mathscr{H}(\lambda)=\mathscr{H}^{*}(\lambda), \quad \int_{-\infty}^{\infty} \frac{\|\mathscr{H}(\lambda)\|}{1+\lambda^{2}} d \lambda<\infty, \quad \operatorname{Im} z>0
$$

а $U$ является постоянной унитарной матрищей. 
ДокАЗАТЕЛЬСтво. Учитывая формулу (2.33) и переходя к пределу в (2.40) при $R \rightarrow \infty$, имеем

$$
\operatorname{det}\left[\frac{1}{2 \pi} \mathscr{P}^{-1}(i) \mathscr{P}^{*-1}(i)\right] \geqslant \exp \frac{1}{\pi} \int_{-\infty}^{\infty} \frac{\ln \operatorname{det} \tau^{\prime}(\lambda)}{1+\lambda^{2}} d \lambda .
$$

Из последнего неравенства вытекает, что $\mathscr{P}^{-1}(\lambda)$ является максимальной матрицей-функцией, удовлетворяющей условию (3.1) (см. [7]). Тогда по теореме Ю. П. Гинзбурга $[8,9]$ справедливо представление (3.12). Теорема доказана.

Из формулы (3.12) и теоремы о предельных значениях мультипликативного интеграла [10] вытекает следующее утверждение.

СлЕДСТВИЕ 3.1. Почти для всех $\lambda \in(-\infty, \infty)$ существуют предельнье значения

и верны формульг

$$
\mathscr{P}_{ \pm}(\lambda)=\lim _{\varepsilon \rightarrow \pm 0} \mathscr{P}(\lambda+i \varepsilon)
$$

$$
\begin{aligned}
& \sqrt{2 \pi} \mathscr{P}_{ \pm}(\lambda)=\lim _{\varepsilon \rightarrow+0}\left[\int_{-\infty}^{\curvearrowleft} \operatorname{le}\right. \exp \left(\frac{1}{2 \pi i} \frac{1+t \lambda}{t-\lambda} \frac{\mathscr{H}(t)}{1+t^{2}} d t\right) \\
&\left.\times e^{ \pm \mathscr{H}(\lambda) / 2} \int_{\lambda+\varepsilon}^{\infty} \exp \left(\frac{1}{2 \pi i} \frac{1+t \lambda}{t-\lambda} \frac{\mathscr{H}(t)}{1+t^{2}} d t\right)\right] .
\end{aligned}
$$

СлЕДСтвИе 3.2. Почти при всех $\lambda$ матрищь $2 \pi \mathscr{P}_{+}^{*}(\lambda) \mathscr{P}_{+}(\lambda) \quad u$ exp $\mathscr{H}(\lambda)$ унитарно эквивалентны.

Действительно, в силу формулы (3.13) имеем

$$
\begin{aligned}
2 \pi \mathscr{P}_{+}^{*}(\lambda) \mathscr{P}_{+}(\lambda)=\lim _{\varepsilon \rightarrow+0}\left\{\left[\int_{\lambda+\varepsilon}^{\infty} \exp \left(\frac{1}{2 \pi i} \frac{1+t \lambda}{t-\lambda} \frac{\mathscr{H}(t)}{1+t^{2}} d t\right)\right]^{-1}\right. \\
\left.\times e^{\mathscr{H}(\lambda)}\left[\int_{\lambda+\varepsilon}^{\infty} \exp \left(\frac{1}{2 \pi i} \frac{1+t \lambda}{t-\lambda} \frac{\mathscr{H}(t)}{1+t^{2}} d t\right)\right]\right\} .
\end{aligned}
$$

Из следствия 3.2 и равенства (3.1) выводим следующее утверждение.

СледствиЕ 3.3. Почти при всех $\lambda$ матрицы $\tau^{\prime}(\lambda)$ и $e^{-\mathscr{H}(\lambda)}$ унитарно эквивалентны.

В силу следствия 3.3 выполняется равенство

$$
\ln \operatorname{det} \tau^{\prime}(\lambda)=-\operatorname{Tr} \mathscr{H}(\lambda), \quad-\infty<\lambda<\infty .
$$

Если $m=1$, то

$$
\ln \tau^{\prime}(\lambda)=-\mathscr{H}(\lambda),
$$

т. е. формула (3.12) принимает вид

$$
\sqrt{2 \pi} \mathscr{P}(z)=e^{i \alpha} \exp \left[\int_{-\infty}^{\infty} \frac{1}{2 \pi i} \frac{1+\lambda z}{z-\lambda} \frac{\ln \tau^{\prime}(\lambda)}{1+\lambda^{2}} d \lambda\right],
$$

где $\alpha=\bar{\alpha}$.

Формула типа (3.14) содержится в статье Крейна [1]. 


\section{ЛИТЕРАТУРА}

1. Крейн М. Г. Континуальные аналоги предложений о многочленах, ортогональных на единичной окружности. ДАН СССР, 105, № 4, 637-640 (1955).

2. Sakhnovich L. A. On one class of canonical systems on half-axis. Integral Equations Operator Theory, 31, No. 1, 92-112 (1998).

3. Sakhnovich L. A. Spectral Theory of Canonical Differential Systems. Method of Operator Identities. Operator Theory, Advances and Applications, vol. 107, Birkhäuser Verlag, 1999.

4. Потапов В. П. Мультипликативная структура $j$-нерастягивающих матрицфункций. Труды ММО, 4, 125-236 (1955).

5. Левитан Б. М. Обратные задачи Штурма-Лиувилля. Наука, М., 1984.

6. Привалов И. И. Граничные свойства аналитических функций. ГИТТЛ, М.-Л., 1950.

7. Розанов Ю. А. Стационарные случайные процессы. Физматгиз, 1963.

8. Гинзбург Ю. П. Мультипликативные представления оператор-функций ограниченного вида. УМН, 22, вып. 1, 163-165 (1967).

9. Ginzburg Yu. P., Shevchuk L. V. On the Potapov theory of multiplicative representations. In: Operator Theory, Advances and Appl., vol. 72, Birkhäuser Verlag, 1994, pp. $28-47$.

10. Сахнович Л. А. Диссипативные операторы с абсолютно непрерывным спектром, Труды МMO, 19, 211-270, (1968). 